Meta

Journal des traducteurs

Translators' Journal

\title{
La traductologie appliquée à la traduction automatique
}

\section{Monique Chevalier, Pierre Isabelle, François Labelle et Claude Lainé}

Volume 26, numéro 1, mars 1981

L'informatique au service de la traduction

Machine Aids to Translation

URI : https://id.erudit.org/iderudit/002229ar

DOI : https://doi.org/10.7202/002229ar

Aller au sommaire du numéro

Éditeur(s)

Les Presses de l'Université de Montréal

ISSN

0026-0452 (imprimé)

1492-1421 (numérique)

Découvrir la revue

Citer cet article

Chevalier, M., Isabelle, P., Labelle, F. \& Lainé, C. (1981). La traductologie appliquée à la traduction automatique. Meta, 26(1), 35-47.

https://doi.org/10.7202/002229ar d'utilisation que vous pouvez consulter en ligne. 


\title{
La traductologie appliquée à la traduction automatique
}

\author{
Monique Chevalier \\ Pierre Isabelle \\ FRANÇOIS LABELLE \\ Claude lainé
}

PRÉAMBULE *

La traduction, tout comme la parole ou la rédaction, est une «opération», une "activité" linguistique. Pour ce qui est des opérations linguistiques unilingues, on note que tout membre d'une communauté linguistique est normalement en mesure de produire et d'interpréter des énoncés dans sa langue. Cette capacité d'interpréter, de «comprendre» des énoncés implique, entre autres choses, la possibilité de mesurer l'équivalence de deux énoncés dans une situation donnée.

Il va de soi que tout sujet bilingue sera capable d'effectuer ces opérations dans chacune des deux langues A et B. Ce qui doit surtout nous frapper, c'est qu'un tel sujet peut en général se prononcer sur le degré d'équivalence entre un énoncé en langue $A$ et un énoncé en langue $B$ dans une situation donnée. Il s'agit alors d'une opération de traduction.

La linguistique se situe évidemment à un niveau différent de celui de la parole ou de la rédaction. La linguistique n'est pas une «opération " linguistique mais plutôt une science ayant pour objet l'analyse des activités linguistiques. Comment maintenant désigner la science ayant pour objet l'analyse des opérations linguistiques de traduction? Certains prêtent au terme «traduction» ce sens additionnel d'activité scientifique. D'autres comme Mounin [5] et Catford [1] parlent d'une «théorie linguistique de la traduction», Nida [6], lui, parle d'une "science de la traduction". Vinay et Darbelnet [7] emploient souvent: «stylistique comparée» dans ce sens.

Quant à B. Harris [3], il propose d'introduire le terme «traductologie» pour désigner cette science de la traduction, et nous retiendrons ce terme. Harris fait remarquer que la traductologie ne doit pas être confondue avec les techniques et les normes apprises par ceux qui deviennent traducteurs professionnels (pas plus qu'on ne confond la linguistique avec les techniques normatives de rédaction). La linguistique et la traductologie sont essentiellement descriptives plutôt que normatives.

* La matière du préambule a été tirée d'un article beaucoup plus élaboré de Pierre Isabelle, «Traductologie et modèles de traduction», qui paraîtra prochainement. 
La plupart des linguistes s'entendent pour dire que la linguistique a pour objet la construction de modèles représentant la compétence du locuteur à produire et interpréter des énoncés dans sa langue. Nous proposons ici de définir de la même façon l'objet de la traductologie, à savoir la construction de modèles représentant la compétence du locuteur bilingue à mesurer l'équivalence d'énoncés appartenant à deux langues naturelles différentes.

La théorie linguistique générale fournit un cadre à l'intérieur duquel chaque langue particulière pourra être décrite. La traductologie générale fournit le cadre à l'intérieur duquel on pourra formuler la description systématique des équivalences entre deux langues données. En réalisant un «modèle linguistique» de traduction sur ordinateur, la traduction automatique constitue une application directe de la traductologie.

\section{LE MODÈLE TAUM}

Le Groupe de recherche en traduction automatique de l'Université de Montréal (TAUM) a réalisé jusqu'ici, sur le même modèle, deux systèmes dont le premier, TAUM/METEO [2], traduit depuis 1977, de l'anglais au français, les prévisions du Centre météorologique canadien. Le second, le système TAUM/AVIATION [4], fait l'objet des recherches du Groupe TAUM depuis le ler avril 1976. Ce dernier est destiné à traduire automatiquement, de l'anglais au français, des manuels d'entretien des circuits hydrauliques d'un avion.

Nous voudrions, dans le présent article, décrire la tâche du spécialiste en traductologie, le traductologue, au sein d'une équipe de traduction automatique comme celle de TAUM. Cette description sera suivie d'une traductionmachine commentée.

\section{Présentation générale du système TAUM/AVIATION}

Il s'agit d'un système de traduction entièrement automatisé conçu pour traduire les textes d'un sous-domaine technique précis: l'hydraulique en aéronautique ${ }^{1}$. La traduction s'effectue, phrase par phrase ${ }^{2}$, en trois grandes étapes: l'analyse, le transfert et la génération.

ANALYSE

Donne une représentation de la phrase contenant l'information essentielle pour la traduction.

\section{TRANSFERT}

Substitue, aux termes anglais, les équivalents français et effectue certaines restructurations des phrases.

La phase d'analyse prépare la phrase pour la traduction en la représentant sous une forme hiérarchisée dans laquelle les unités lexicales sont accompa-

1. Ce n'est pas un système de traduction «universel».

2. On ne tient donc pas compte des relations entre phrases. 
gnées d'une description des phénomènes susceptibles de conditionner leur traduction.

À la phase de transfert, l'ordinateur consulte un dictionnaire bilingue et substitue aux unités lexicales anglaises les équivalents français. Il effectue également des restructurations pouvant être exigées par le choix de certaines unités lexicales françaises.

Enfin, la génération reproduit la phrase selon les règles de la syntaxe et de la morphologie du français.

\section{Préparation de la phrase pour la traduction}

Nous savons tous que la plupart des mots d'une langue ont plusieurs sens possibles - donc plusieurs équivalents possibles dans une autre langue - et que le choix de l'équivalent approprié dépend essentiellement du contexte. Ainsi, dans la phrase (1), le verbe anglais service peut se traduire par «remplir» ou par «faire l'entretien de» selon que son objet direct désigne ou non un «contenant $\gg$ :

(1) The $\left\{\begin{array}{l}\text { tank } \\ \text { aircraft }\end{array}\right\}$ should be serviced by personnel.

La nature sémantique de l'objet direct de service est un facteur pertinent pour la traduction de ce verbe. Par contre, les unités lexicales the, by, ou personnel n'influencent pas la traduction de service.

S'il est banal, pour un traducteur, de repérer l'objet direct d'un verbe, il n'en va pas de même pour l'ordinateur car rien ne lui indique que tank est l'objet direct plutôt que the ou personnel. La tâche de l'analyse consiste précisément à préparer la phrase pour les opérations de traduction en déterminant les relations grammaticales entre les mots d'une phrase et à inclure dans la représentation structurale de cette phrase tous les éléments pertinents à la traduction. Ainsi, la phrase (1) aura la représentation simplifiée suivante :

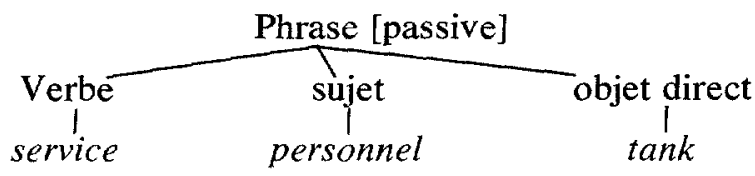

Toutes les phrases sont représentées sous une telle forme schématisée. Le traductologue doit alors écrire un algorithme (séquence d'instructions) qui, à partir de telles représentations, repérera les facteurs gouvernant la traduction de chaque unité lexicale. Dans le cas de service par exemple, la nature sémantique de l'objet direct est un facteur à repérer alors que, pour un autre verbe, ce pourrait être la nature du sujet.

Pour construire, à partir de la phrase (1), une représentation du type (2), l'ordinateur consulte d'abord le dictionnaire d'analyse qui contient l'information suivante sur les unités lexicales anglaises:

- personnel nom de type sémantique [humain] 
- tank
- service

nom de type [concret, pièce, contenant]

verbe dont le sujet possède le trait [humain] dont l'objet direct doit posséder le trait [pièce] et qui peut également avoir un objet indirect introduit par la préposition with (ex : service reservoir with one gallon of oil). Ces traits sémantiques sont des propriétés combinatoires de service : ils déterminent le type de sujet ou d'objet que peut accepter ce verbe (ce que nous appelons sa complémentation). Les traits sémantiques de personnel ou tank sont plutôt des traits inhérents.

L'ordinateur parcourt ensuite la phrase de gauche à droite en tentant d'identifier les relations grammaticales entre les mots de la phrase. Ainsi, le premier nom tank, sera retenu comme sujet possible. Cependant, à la lecture du verbe service, cette hypothèse sera rejetée puisque le sujet doit être de type [humain]. De plus, la présence de is et la terminaison -ed indiquent qu'il s'agit d'une phrase passive. Tank sera alors considéré comme l'objet direct, ce qui est en accord avec la complémentation de service ${ }^{3}$. Il reste ensuite à rechercher le sujet manquant - personnel - et à construire la représentation de la phrase.

$\mathrm{Si}$, au lieu de la phrase (1) on avait eu la phrase (3), nous n'aurions obtenu aucune analyse car, d'après la complémentation de service au dictionnaire d'analyse, ce verbe n'admet qu'un objet direct possédant le trait [pièce].

(3) The public should be serviced by personnel.

D'après les textes d'hydraulique que nous avons dépouillés pour élaborer le système TAUM/AVIATION, service n'apparait pas avec un objet direct de type [humain]. En ce sens, la phrase (3) est considérée, pour le système actuel, comme une phrase «impossible».

En résumé, l'information contenue au dictionnaire d'analyse indique quelles sont les phrases acceptables pour le système en déterminant soit le type d'entité qu'un terme peut désigner (par exemple, pour TAUM/AVIATION, tank peut désigner un [contenant] mais non un [véhicule]), soit le type de sujet et d'objet direct avec lesquels un verbe ou un adjectif peuvent se combiner. En d'autres mots, le dictionnaire d'analyse détermine, dans le cadre du souslangage traité par TAUM/AVIATION, l'ensemble des contextes possibles d'une unité lexicale. C'est à partir de ces données de base que s'élabore le travail du traductologue.

\subsection{Le rôle du traductologue}

Du point de vue de la traduction, chaque contexte possible pour une unité lexicale pourrait, théoriquement, déterminer le choix d'un équivalent différent dans l'autre langue. Ainsi, dans le cas de service trois des contextes possibles prévus par le dictionnaire d'analyse déterminent la traduction de service :

3. Le choix entre les homographes se fait à l'analyse; bien qu'en général, service puisse être employé comme nom, cette possibilité est à exclure en 1 , sinon la phrase n'aurait pas de verbe. 
(4) a) si l'objet direct est un [contenant], traduire service par «remplir»;

b) si l'objet direct n'est pas un [contenant] traduire service par «faire l'entretien de»;

c) s'il y a un objet indirect introduit par with, traduire service par «mettre».

ex : a) Service tank with one gallon of oil.

b) Mettre un gallon d'huile dans le réservoir.

Mais il arrive également qu'une unité lexicale n'ait qu'un seul équivalent, quel que soit le contexte; c'est le cas, par exemple, de red qui se traduira par "rouge», quelle que soit la nature de son sujet (du moins dans le sous-langage que nous traitons).

Le traductologue doit examiner pour chaque unité lexicale, l'ensemble de ses contextes possibles d'après le dictionnaire d'analyse et décider, pour chaque contexte, quel est l'équivalent approprié. Il doit ensuite écrire, dans un langage de programmation spécialisé, une séquence d'instructions comportant des tests sur les contextes pertinents et des opérations de traduction, semblables en gros à (4) a), b) et c).

Ces opérations de traduction peuvent parfois être plus complexes que celles qu'on retrouve en (4) a), b), c), où elles se réduisent à une simple substitution de l'équivalent approprié. Ainsi, par exemple, dans l'entrée du verbe arise, le traducteur a décidé que, lorsque ce verbe est suivi d'un objet indirect introduit par from, il se traduit par "produire" (ex. (5). La règle traduisant arise par «produire» ne saurait consister en une simple substitution; elle entraine également la permutation du sujet et de l'objet :

(5A) Leakage may arise from excessive pressure in the system.

(5F) Une surpression dans le circuit peut produire une fuite.

En résumé, les entrées écrites par le traductologue au dictionnaire de transfert consistent en une série de tests portant sur le contexte de l'unité à traduire, suivis d'opérations de traduction allant de la simple substitution de l'équivalent approprié à la modification de l'environnement de l'unité traduite.

Le système TAUM/AVIATION repose sur une hypothèse intéressante du point de vue de la théorie de la traduction: nous croyons, à la lumière de notre expérience, qu'il est possible d'obtenir une traduction fiable et aisément compréhensible à partir d'une représentation de l'environnement local des unités à traduire et en utilisant une formalisation des concepts grammaticaux traditionnels tels que : sujet, objet direct, objet indirect, etc.

Nous venons de décrire de façon sommaire le travail d'écriture des entrées au dictionnaire de transfert. Nous allons maintenant décrire en détail l'entrée du verbe consider qui présente plusieurs opérations typiques.

\subsection{Exemple d'entrée de dictionnaire}

Le rôle du dictionnaire d'analyse est donc d'établir pour chacune des unités lexicales anglaises, l'ensemble des contextes d'utilisation de ces unités 
dans le sous-langage traité. Nous avons à l'Annexe 1 l'entrée du verbe consider telle qu'elle a été formalisée au dictionnaire d'analyse. Cette formalisation du verbe consider représente les différents usages de ce mot que nous avons recensés dans les manuels d'hydraulique. Les phrases (6) à (15) font donc partie de la classe de phrases décrites par la complémentation du verbe consider.

(6) Consider the damage as extending to the wing tip.

(7) Accuracy is considered as the most important criterion.

(8) Consider these areas for repair.

(9) Consider whether the damage is negligible.

(10) Consider cleaning the filter.

(11) The system is considered serviceable.

(12) Consider the damage to extend to the wing tip.

(13) Consider that any increase in turbine RPM results in overtemperature.

(14) Consider the direction of prevailing winds.

(15) Consider each cylinder to make sure scratches are negligible.

À partir de cette énumération des divers usages du verbe consider, le traductologue doit trouver des tests qui permettront à l'ordinateur de choisir les bons équivalents français ${ }^{4}$.

L'entrée au dictionnaire d'analyse nous indique que le sujet du verbe consider n'est que [humain]. Le sujet n'est donc pas un facteur discriminant pour traduire le verbe. La traduction de consider dépendra plutôt de la nature de son objet direct ou de son objet indirect. Les objets direct et indirect peuvent être soit un groupe nominal, soit une proposition :

1. Si l'objet indirect de consider est une proposition gérondive introduite par as, traduire consider par «supposer que» et transformer l'objet direct de consider (damage) en sujet de la gérondive (extend) :

(6A) Consider the damage as extending to the wing tip.

(6F) Supposer que les dommages s'étendent jusqu'au saumon d'aile.

2. Si l'objet indirect est un groupe nominal, il peut être introduit par as ou for:

a) si le groupe nominal est introduit par as, traduire consider par «considérer»:

(7A) Accurary is considered as the most important criterion.

(7F) La précision est considérée comme le critère le plus important.

b) si le groupe nominal est introduit par for, traduire par «envisager», effacer for; transformer l'objet direct (areas) en complément de l'objet indirect (repair); enfin, déplacer l'objet indirect en objet direct :

4. On trouvera à l'Annexe 2 l'entrée du verbe consider telle qu'elle a été formalisée au dictionnaire de traduction. 
(8A) Consider these areas for repair.

(8F) Envisager la réparation de ces zones.

Nous venons d'examiner différents types d'objet indirect qui déterminent la traduction de consider. Lorsque l'objet indirect est absent, l'objet direct de consider peut servir à l'élaboration des tests de traduction. L'objet direct peut être lui aussi une proposition ou un groupe nominal.

3. Si l'objet direct est une proposition, on doit distinguer les cas suivants :

a) si la proposition est une question indirecte, traduire consider par «déterminer» :

(9A) Consider whether the damage is negligible.

(9F) Déterminer si les dommages sont négligeables.

b) si la proposition est une gérondive, traduire consider par «penser à»; le changement de la forme -ing en infinitif sera fait automatiquement par une règle générale :

(10A) Consider cleaning the filter.

(10F) Penser à nettoyer le filtre.

c) si la proposition est adjectivale, traduire par «considérer»; le changement de proposition réduite en proposition pleine est noté dans l'entrée de dictionnaire et sera exécuté automatiquement par une règle générale :

(11A) The system may be considered serviceable.

(11F) On peut considérer que le circuit est utilisable.

d) si la proposition est une infinitive, traduire par «supposer que"; le changement de l'infinitive en phrase à temps fini se fera automatiquement :

(12A) Consider the damage to extend to the wing tip.

(12F) Supposer que les dommages s'étendent jusqu'au saumon d'aile.

e) si la proposition est introduite par that, traduire par «supposer»:

(13A) Consider that any increase in turbine RPM results in overtemperature.

(13F) Supposer que toute augmentation dans le régime de la turbine entraîne une surchauffe.

4. L'objet direct peut également être un groupe nominal : il y a alors deux possibilités :

a) si le groupe nominal est du type sémantique [abstrait], traduire consider par «tenir compte de»:

(14A) Consider the direction of prevailing winds.

(14F) Tenir compte de la direction des vents dominants.

b) si le groupe nominal est du type sémantique [pièce], traduire par «examiner»: 
(15A) Consider each cylinder to make sure scratches are negligible.

(15F) Examiner chaque cylindre pour s'assurer que les éraflures sont négligeables.

Nous avons voulu illustrer comment, avec des tests portant uniquement sur l'environnement immédiat de l'unité lexicale (dans notre exemple, objets direct et indirect) nous pouvons arriver à une traduction assez fidèle et, dans tous les cas, très compréhensible.

Nos tests ont été élaborés à partir d'un corpus fini de phrases (cf. (6) à (15) appartenant à la classe des phrases décrites par la complémentation de consider. À partir de cette complémentation, nous avons essayé d'atteindre la plus grande généralisation possible c'est-à-dire de couvrir non seulement des phrases qu'on a vues, mais l'ensemble des phrases possibles. Par exemple, notre algorithme de traduction du verbe consider rend compte des phrases (16) à (18) où consider a un complément d'objet direct de type adjectival. Consider sera traduit par «considérer» comme en (11).

(16) A measured contaminant level should be considered acceptable.

(17) The landing gear should be considered satisfactory if inspection after flight shows only that a seepage of fluid has collected.

(18) Battery starts at $-54^{\circ} \mathrm{C}$ are considered satisfactory.

Toutefois, les mécanismes de production linguistique sont très diversifiés. Compte tenu de cette grande richesse de production propre à toute langue naturelle, nous pourrons souvent trouver un contre-exemple à toute généralisation.

\section{Exemple de traduction}

Nous allons maintenant commenter la traduction-machine d'un corpus constitué de phrases tirées d'un manuel de maintenance en hydraulique. Les phrases choisies nous permettront de faire ressortir certaines transformations que le lecteur attentif saura relever - ainsi que certaines erreurs qui, dans bien des cas, sont le reflet direct des limites du système, limites dont le traductologue doit tenir compte lors de l'écriture des entrées.

"A manually adjustable pressure compensator must be used to adjust the outlet pressure. Cap all lines leading to system being tested. Check the locking mechanism of the landing gear doors. If the system fails, a hydraulic hand pump can be used to open the doors. If caution light fails to go off, set pump control switch to OFF position. If pressure builds up in a component, the packing tries to move in its groove. If it moves, it tends to roll, causing a slight pumping action. Release all pressure from system. Analyze hydraulic fluid samples, using 644-2 contamination analysis kit. Resample hydraulic fluid from reservoir of the affected system in accordance with paragraph No. 1. The valve opens when it is acted upon by a force opposed to the spring pressure. Hydraulic fluid enters the inlet-coupling and is passed through fin-walled tubing which acts as a heat exchanger.'

\section{TRADUCTION-MACHINE}

«Utiliser un compensateur de pression réglable manuellement pour régler la pression de sortie (1). Obturer toutes les canalisations menant 
au circuit qu'on vérifie. Vérifier le mécanisme de verrouillage des trappes du train d'atterrissage. Si le circuit tombe en panne, une pompe hydraulique de la main (2) peut être utilisée pour ouvrir les portes*(3). Si le voyant d'avertissement ne s'éteint pas, placer le commutateur de la pompe à la position OFF. Si la pression augmente dans un composant, la garniture a tendance à bouger dans sa gorge. S'il (4) bouge, il (4) a tendance à rouler, causant une légère action de pompage. Libérer (5) toute la pression du circuit. Analyser les échantillons de liquide hydraulique à l'aide de la trousse d'analyse de contamination 644-2. Rééchantillonner le liquide hydraulique du réservoir du circuit concerné conformément au paragraphe no. 1 . Le clapet ${ }^{*}(6)$ s'ouvre lorsqu'il est soumis à une force opposée à la pression du ressort. Le liquide hydraulique pénètre dans le raccord d'entrée et passe par le tuyau à ailettes qui joue le rôle d'un échangeur de chaleur.»

Nous avons vu précédemment que le traductologue ne dispose que des informations syntaxico-sémantiques contenues dans un macrocontexte - la phrase. Il ne peut donc désambiguïser un terme à l'aide d'un co-référent situé dans un contexte plus large (plus d'une phrase). C'est le cas en (3) où door est rendu par "porte» alors que dans la phrase précédente il est traduit par «trappe». Lors de l'écriture de cette entrée, le traductologue a cherché à exploiter le microcontexte - environnement immédiat - de door pour distinguer «trappe» de «porte». Il a pallié l'ignorance de l'ordinateur dans le domaine en faisant le test ponctuel suivant : si door figure dans l'un des groupes nominaux landing gear door ou bomb bay door alors traduire door par «trappe», sinon traduire par "porte». Dans le dernier cas, il a inséré un symbole - ici un astérisque pour signaler au réviseur son incapacité de prévoir à tout coup la bonne traduction. Nous avons le même type de problème en (4) où le pronom - masculin par défaut - réfère en fait au nom féminin «garniture» de la phrase précédente - incapacité de traduire correctement les pronoms anaphoriques.

L'erreur (6) est également liée au manque de contexte. Le terme polysémique valve a entre autres équivalents possibles «soupape, clapet, robinet, vanne, valve ... ». La traduction de ce terme présente peu de difficultés pour le traducteur qui fait intervenir contexte et connaissances techniques. Mais l'ordinateur, lui, ne dispose que d'un contexte restreint. Par conséquent, le traductologue ne peut que proposer l'équivalent - accompagné d'un symbole de révision - qu'il juge le plus général dans le domaine concerné.

Également attribuable au manque de connaissance du monde, l'erreur (1) illustre en plus la difficulté de saisir les relations entre les constituants d'un groupe nominal. Nous avons ici un glissement de sens où outlet pressure, "pression à la sortie» est confondu avec output pressure, "pression de sortie" - ces deux groupes nominaux ont exactement la même représentation et les mêmes traits. En effet, la «pression à la sortie» se mesure à la sortie de la pompe alors que la «pression de sortie» peut se mesurer plus haut dans le circuit. On voit qu'une connaissance des relations sémantiques entre les constituants de ce groupe nominal ne suffirait pas, il faut faire intervenir des connaissances techniques.

Par contre, une bonne connaissance des relations sémantiques entre constituants permettrait d'éviter l'erreur (2). À ce stade, on connaît malheureusement 
peu de choses sur ces relations et un rapide examen des groupes nominaux ci-dessous ayant tous un point commun - nom concret précédé d'un nom à caractéristique humaine - permet d'apprécier l'ampleur de la tâche, soit : eye protective equipment, crew compartment, face mask, pilot's control stick. En français, les relations entre constituants de ces groupes nominaux, s'expriment toutes par une charnière différente. Et, il y a même dans un cas effacement de l'un des constituants - face mask, "masque». Ce problème déborde actuellement le rôle principal du traductologue et la solution devra s'inscrire dans un traitement global reposant sur une étude sémantique.

En (5), on a délibérément choisi de traduire release the pressure par «libérer la pression». On aurait pu préférer «dépressuriser» pour des raisons d'idiomaticité technique. Voyons toutefois ce qui a guidé le traductologue dans son choix. La phrase release all pressure from system est représentée à l'analyse de la façon suivante :

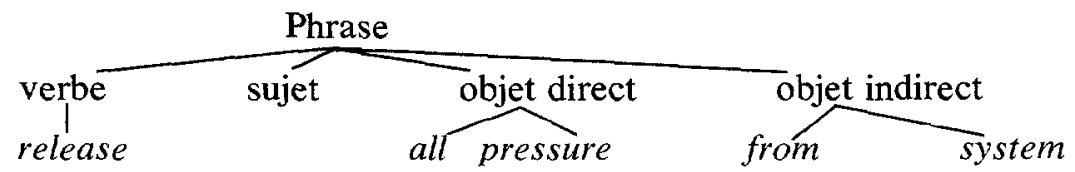

Si l'on traduit par «dépressuriser complètement le circuit», il faut : effacer pressure et from, déplacer system en objet direct et rattacher all au verbe. Ce qui donne :

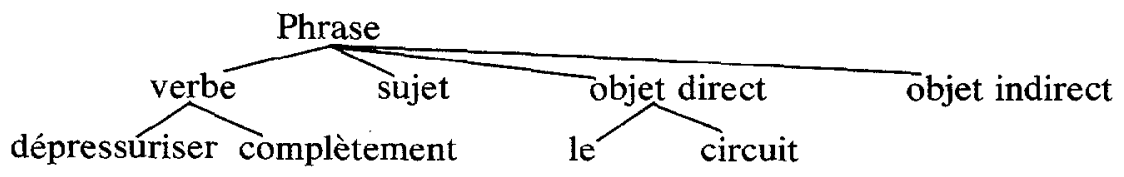

On pourrait effectuer cette restructuration mineure chaque fois que l'objet direct de release est une variable. Mais, poursuivons l'examen avec la phrase suivante : release all residual pressure left in the system.

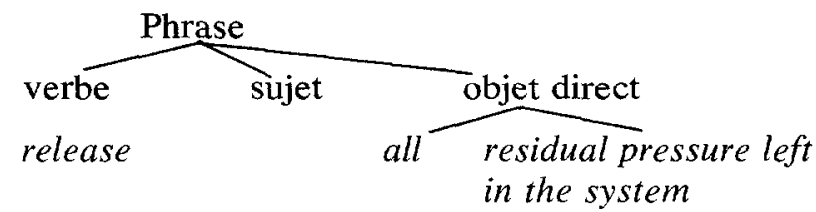

À moins de prévoir un test supplémentaire qui bloquerait la restructuration chaque fois que l'objet direct est modifié, celle-ci va s'appliquer, puisque l'objet direct de release est une variable, et nous obtiendrons «dépressuriser complètement... résiduel laissé dans le circuit». Par conséquent, ne pouvant prévoir tout ce qui, dans ce cas, peut se greffer à l'objet direct, le traductologue a préféré retenir une traduction générale plutôt que de risquer de générer un non-sens.

Ce rapide examen d'une traduction-machine permet de faire ressortir les contraintes imposées au traductologue par le système et également, la réflexion que ce dernier s'impose lors de l'écriture de chaque entrée. La traduction obtenue, bien que très littérale, est fidèle au texte de départ, facilement révisable et exempte d'erreur majeure. 
Les résultats actuels obtenus en traduction automatique sont des plus encourageants. Nous espérons, surtout après le succès total de TAUM/MÉTÉO, que la traduction automatique sera perçue par le traducteur comme un outil qui pourrait le dispenser presque complètement des travaux monotones où son bagage de connaissances n'est pas mis à profit.

Le traductologue a un rôle important à jouer. Il peut faire avancer les connaissances en linguistique contrastive par la découverte et la formalisation des mécanismes régissant le passage d'une langue à une autre. Pour ce qui est du traducteur, c'est à lui qu'il appartient surtout de découvrir et de suggérer des domaines d'application pour la traduction automatique.

\section{BIBLIOGRAPHIE}

1. CATFORD, J. C., A Linguistic Theory of Translation, Oxford University Press, 1965.

2. CHEVALIER, M., J. DANSEREAU, G. POULIN, TAUM/MÉTÉO: description du système, TAUM, Université de Montréal, janvier 1978.

3. HARRIS, B., «La traductologie, la traduction naturelle, la traduction automatique et la sémantique", in Cahier de linguistique no. 2, les Presses de l'Université du Québec, Montréal, 1973.

4. ISABELLE, P., L. BOURBEAU, M. CHEVALIER, et S. LEPAGE, TAUM/AVIATION : description d'un système de traduction automatisée des manuels d'entretien en aéronautique, $7 \mathrm{e}$ Congrès international de linguistique computationnelle (COLING), Bergen, Norvège, août 1978.

5. MOUNIN, G., les Problèmes théoriques de la traduction, Paris, Gallimard, 1963.

6. NIDA, E., Towards a Science of Translation, Leiden, Brill, 1964.

7. VINAY, J.-P. et J. DARBELNET, Stylistique comparée du français et de l'anglais, Paris, Didier, 1958.

Nous remercions Marcel Paré, directeur du groupe TAUM, pour ses conseils toujours judicieux. Nous remercions également Monique Boileau, Louise Claude, Lorraine Léonard et Françoise Parc, traducteurs à la section de traduction automatique du Secrétariat d'État du Canada. Le présent article repose sur leur expérience de travail à la phase de transfert du système TAUM/ AVIATION.

\section{ANNEXE 1}

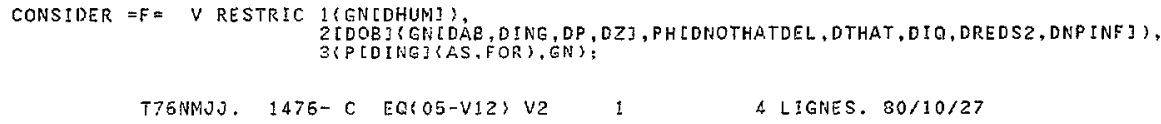




\section{ANNEXE 2}

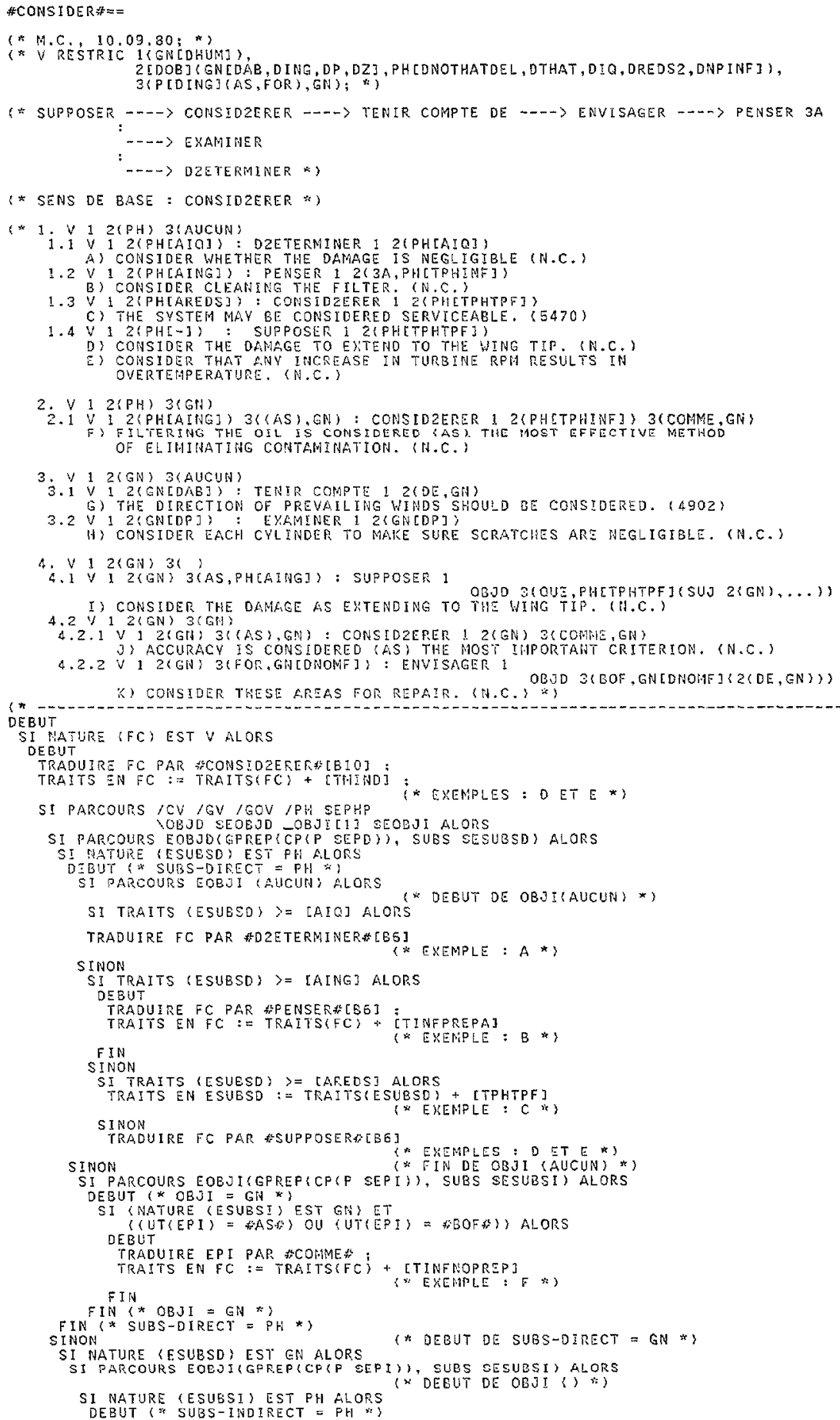




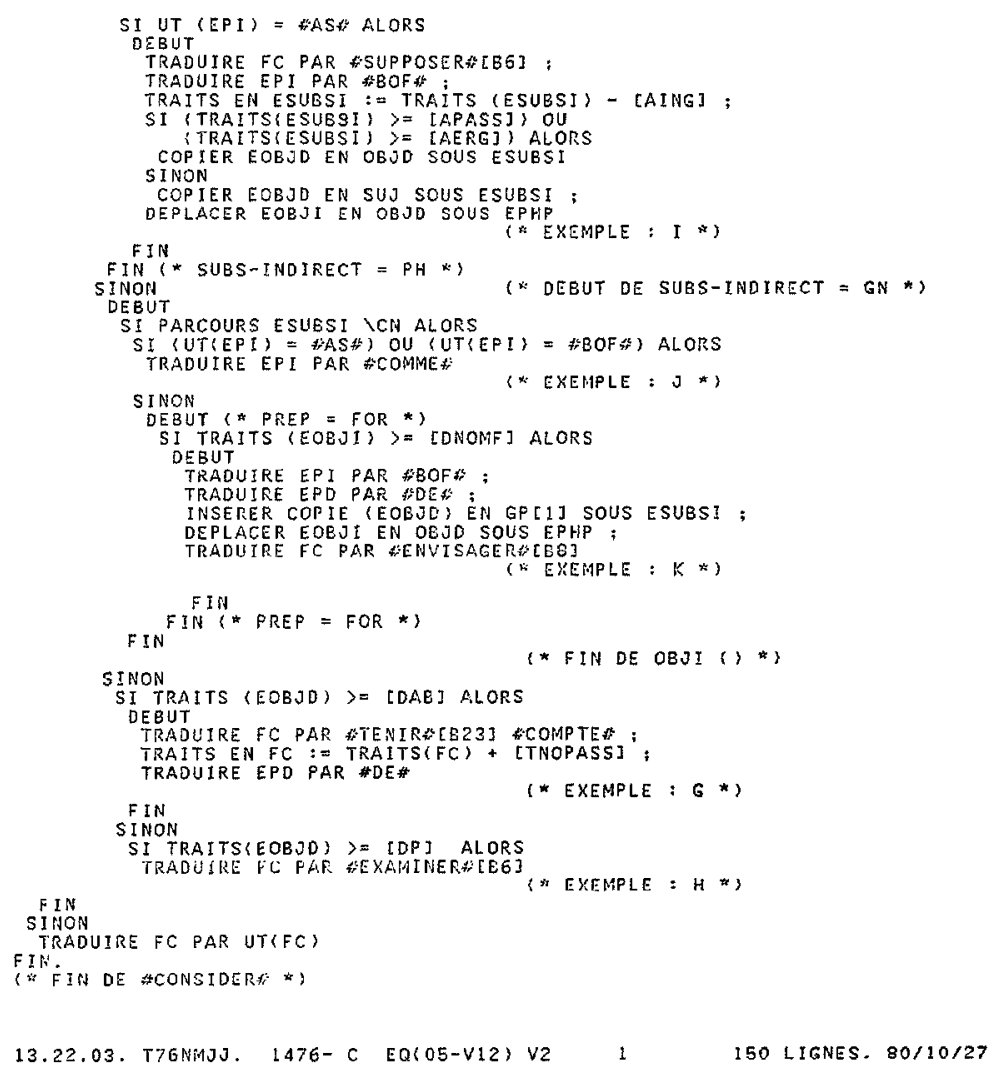

